\title{
The Changing Role for Endomyocardial Biopsy in the Diagnosis of Giant-Cell Myocarditis
}

\author{
Ankur Kalra • Rachel Kneeland · \\ Michael A. Samara • Leslie T. Cooper Jr.
}

To view enhanced content go to www.cardiologytherapy-open.com

Received: February 26, 2014 / Published online: May 9, 2014

(c) The Author(s) 2014. This article is published with open access at Springerlink.com

\begin{abstract}
Endomyocardial biopsy (EMB) is central to the diagnosis of giant-cell myocarditis (GCM) and planning further management. There is, however, no guideline-directed recommendation on re-biopsy or left ventricular EMB in a suspected case of acute, fulminant myocarditis following an indeterminate first biopsy. This manuscript illustrates, with a case, the changing role for $\mathrm{EMB}$ in the current era in the diagnosis of GCM.
\end{abstract}

Electronic supplementary material The online version of this article (doi:10.1007/s40119-014-0028-5) contains supplementary material, which is available to authorized users.

A. Kalra $(\varangle) \cdot$ M. A. Samara

Advanced Heart Failure and Cardiac

Transplantation, Minneapolis Heart Institute at

Abbott Northwestern Hospital, Minneapolis, MN, USA

e-mail: kalramd.ankur@gmail.com

R. Kneeland

Arizona College of Osteopathic Medicine,

Midwestern University, Glendale, AZ, USA

L. T. Cooper Jr.

Cardiovascular Disease, Mayo Clinic, Rochester, MN, USA
Keywords: Cardiology; Myocarditis; Giant cell; Endomyocardial biopsy (EMB); Left ventricular EMB

\section{INTRODUCTION}

Giant-cell myocarditis (GCM) is a rare disease that is characterized by diffuse inflammatory infiltration of the myocardium with lymphocytes and multi-nucleated giant cells in the absence of granulomas [1]. It most commonly presents as heart failure with ventricular arrhythmias and cardiac conduction abnormalities [1]. The clinical course in GCM is typically fulminant, with ensuing death or cardiac transplantation within hours or days of presentation, despite initiation of guideline-directed medical therapy [1]. Endomyocardial biopsy (EMB) is key in establishing the correct diagnosis, and planning further management. There is, however, no guideline-directed recommendation on re-biopsy or left ventricular (LV) EMB in a suspected case of acute, fulminant myocarditis following an indeterminate first biopsy. This manuscript illustrates, with a case, the changing role for EMB in the current era in the diagnosis of GCM. 


\section{CASE REPORT}

Informed consent was obtained from this patient for being included in the paper.

\section{HISTORY}

A 50-year-old male presented to his primary care physician in rural Minnesota (United States) with progressive shortness of breath and functional limitation of 2-month duration, following antecedent viral upper respiratory infection. The patient also reported dizziness, lightheadedness, palpitations, and chest tightness with physical activity. Twenty-four-h ambulatory electrocardiographic (Holter) monitoring demonstrated sustained monomorphic slow ventricular tachycardia (VT) at 106 beats per minute. He was admitted to a local hospital. Cardiac troponin I (cTnI) was elevated at $3.66 \mathrm{ng} / \mathrm{mL}$ (reference $<0.034 \mathrm{ng}$ / $\mathrm{mL}$ ). Brain natriuretic peptide level was $5,500 \mathrm{pg} / \mathrm{mL}$ (reference $4-40 \mathrm{pg} / \mathrm{mL}$ ). A transthoracic echocardiogram showed globally depressed LV systolic function with marked septal dyssynchrony; estimated ejection fraction was $25-0 \%$. Right ventricular systolic function was moderately reduced with biatrial enlargement. There was no significant valvular abnormality or pericardial effusion. The patient was treated with intravenous heparin and amiodarone, and transferred to our tertiary-care center for further evaluation and management.

The past medical history was significant for essential hypertension and hyperlipidemia. The patient was a lifelong non-smoker and reported consumption of 4-6 units of alcohol per day. There was no history of illicit drug use. The family history was significant for chronic granulomatous disease in a brother who died at age 48 of Staphylococcal sepsis. His maternal grandparents had died in their early 1940s of unknown causes. He had never traveled outside the United States. Prescription medications included atenolol, amlodipine, lisinoprilhydrochlorothiazide, and simvastatin.

\section{PHYSICAL EXAMINATION}

On physical examination, the patient's temperature was $37.3^{\circ} \mathrm{C}(99.1 \mathrm{~K})$, his heart rate was irregular at 102 beats per minute, his blood pressure was $102 / 80 \mathrm{mmHg}$, his respiratory rate was 13 breaths per minute, and his oxygen saturation (via pulse oximetry) was $97 \%$ on $3 \mathrm{~L} /$ min on inhalational oxygen via nasal cannula. The jugular venous pressure was elevated at $15 \mathrm{cmH}_{2} \mathrm{O}$. The point of maximal impulse was palpated in the 6 th intercostal space, $3 \mathrm{~cm}$ lateral to the midclavicular line. Heart sounds were distant. There was an S3 gallop with minimal bibasilar crackles on cardiopulmonary auscultation. Hands and feet were warm with intact peripheral pulses. There was trace pedal edema. Review of systems was otherwise unremarkable.

\section{DIAGNOSTIC STUDIES}

Laboratory tests, including a complete blood count and basic metabolic panel, as well as an international normalized ratio and activated partial thromboplastin time were within normal limits. Chest roentgenogram demonstrated a mildly enlarged cardiac silhouette. A 12-lead electrocardiogram (EKG) showed normal sinus rhythm with low-voltage QRS complexes (134 ms), Q waves in leads III \& $\mathrm{aVF}$, poor R-wave progression and evidence of left atrial enlargement. cTnI was elevated at $3.351>2.915 \mathrm{ng} / \mathrm{mL}$ (reference $<0.034 \mathrm{ng} / \mathrm{mL}$ ). 


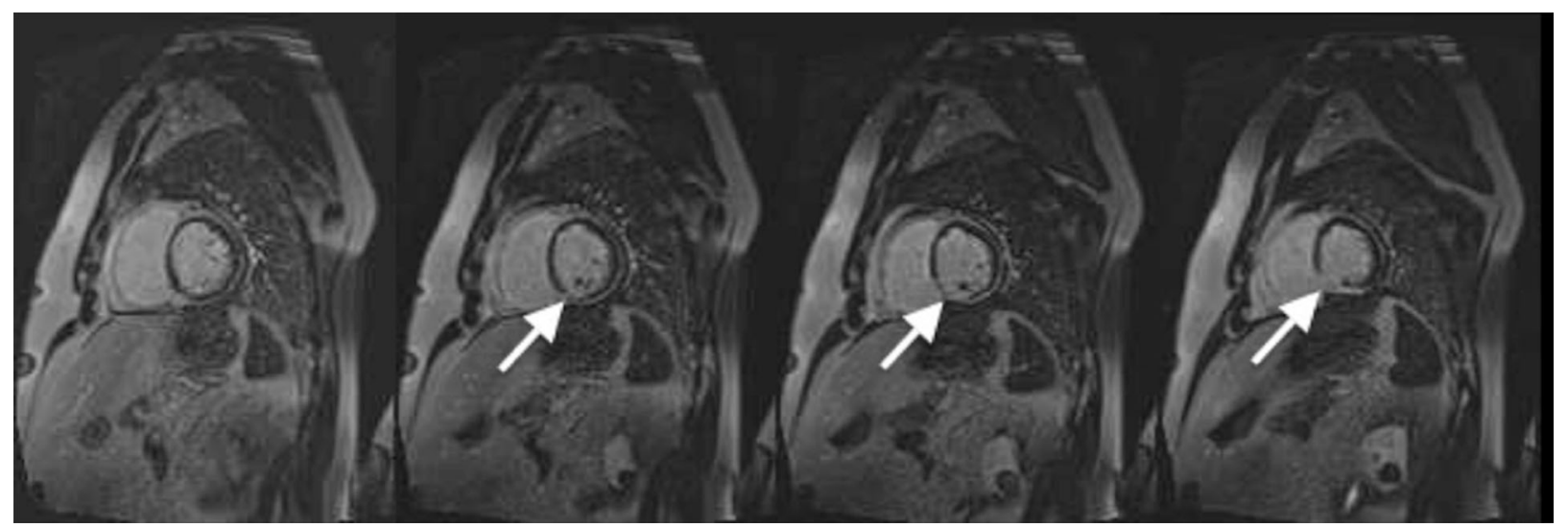

Fig. 1 Cardiac magnetic resonance imaging: T1-weighted images demonstrating delayed hyper-enhancement (arrows) in mid-inferior/septal walls

Coronary angiography revealed minimal nonobstructive epicardial coronary artery disease; the LV end-diastolic pressure was $36 \mathrm{mmHg}$. Right heart catheterization showed elevated right- and left-sided filling pressures and evidence of cardiogenic shock [right atrial pressure of $23 \mathrm{mmHg}$, right ventricular (RV) pressure of $33 / 20 \mathrm{mmHg}$, pulmonary artery pressure of $42 / 34 \mathrm{mmHg}$, mean pulmonary artery pressure of $38 \mathrm{mmHg}$, pulmonary capillary wedge pressure of $23 \mathrm{mmHg}$, and a Fick cardiac index of $\left.1.4 \mathrm{~L} / \mathrm{min} / \mathrm{m}^{2}\right]$. A cardiac magnetic resonance imaging (cMRI) scan with gadolinium demonstrated a mildly enlarged and severely hypokinetic left ventricle with prominent septal dyssynchrony. There was focal delayed hyper-enhancement on T1weighted imaging in the mid-inferior wall and mid-inferior septum (Fig. 1).

An EMB from an MRI-guided site in the RV septum showed extensive fibrotic replacement of the myocardium with associated scattered fatty tissue and limited chronic inflammation. Iron and crystal violet staining were negative for iron and amyloid deposition. There was no serologic evidence of a co-morbid autoimmune disorder. He had sustained slow monomorphic VT, refractory to pace-termination in the cardiac catheterization laboratory. Electrophysiology study demonstrated multiple inducible VTs at variable cycle lengths with extensive low-voltage area in right ventricle and inhomogeneous scar in the inferoseptal and anterolateral walls of left ventricle.

The patient was emergently listed for cardiac transplantation and a pre-transplantation immunosuppressive regimen of methylprednisolone and mycophenolate mofetil was administered. Orthotopic heart transplantation was performed. He succumbed to post-cardiotomy shock requiring vasopressor and inotropic support post-transplantation. Pathology of the explanted heart revealed florid GCM with extensive replacement fibrosis involving the interventricular septum (IVS) (Fig. 2) and the LV free wall. There was acute hemorrhagic infarction of the IVS. Histopathology demonstrated multi-nucleated giant cells with hypereosinophilic and necrotic cardiac myocytes (Fig. 3).

\section{DISCUSSION}

The diagnosis of GCM is established by a conglomerate of laboratory and radiographic 


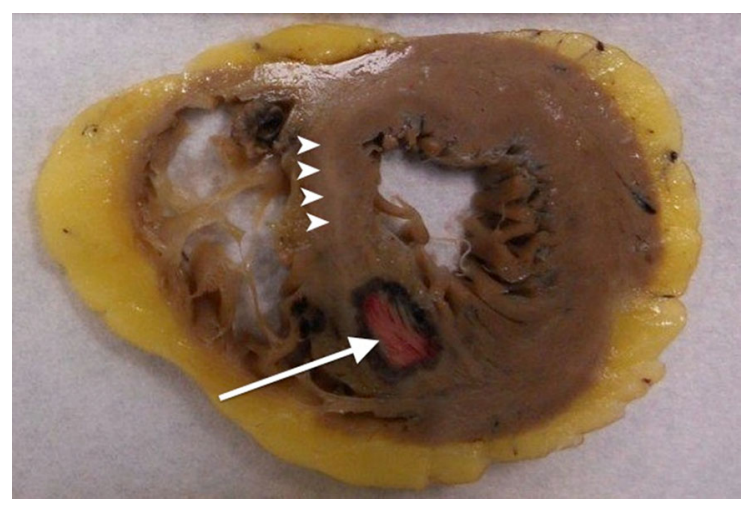

Fig. 2 Extensive replacement fibrosis of the interventricular septum (IVS) (arrowheads) with acute hemorrhagic infarction of the IVS (arrow)

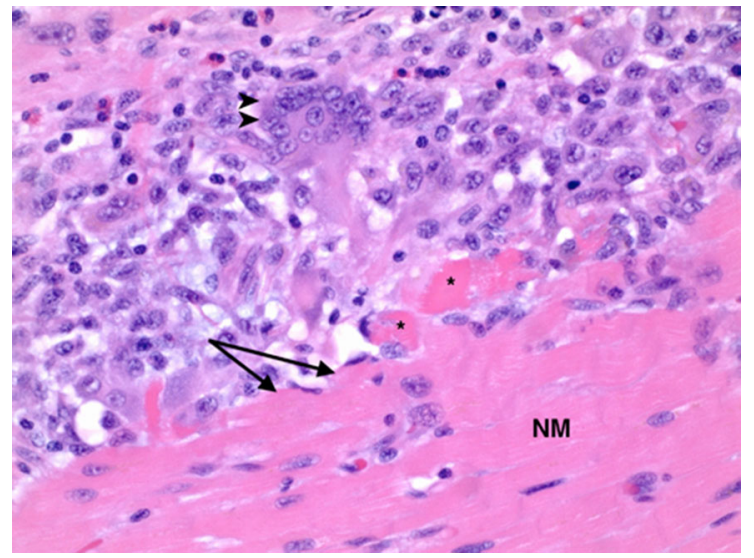

Fig. 3 Interface (arrows) between active giant-cell myocarditis and viable normal myocardium (NM) with hypereosinophilic and necrotic cardiac myocytes (asterisk). Multi-nucleated giant cells (arrowheads) are seen invading into NM

investigations. Biomarkers of cardiac injury, troponin I and $\mathrm{T}$ are typically constitutively elevated, with a lack of rise-and-fall pattern, and reliably predict severity of myocarditis and short-term prognosis [1]. There are no pathognomonic EKG changes that occur in GCM. Electrocardiographic abnormalities include sinus tachycardia and non-specific ST changes, and T-wave abnormalities. Occasionally, patients can present with ST-elevation on the surface EKG, with pathologic Q waves. Presence of pathologic Q waves, and QRS duration >120 ms have been shown to predict an increased risk of death or cardiac transplantation [2]. The most common echocardiographic finding in acute myocarditis is a dilated, spherical left ventricle with reduced systolic function. Concomitant RV dysfunction portends a poor prognosis. cMRI can be a useful tool, and is being utilized with increased frequency, but has not been extensively studied in GCM [3].

Endomyocardial biopsy is central to the diagnosis of GCM, as it is a pathologic diagnosis. The earliest use of EMB was reported by Sakakibara and Konno [4], using the Konno bioptome via the basilic vein and axillary artery. Biopsy specimens were obtained from five patients without complications. In all cases, a substantially more accurate histological diagnosis was obtained via EMB than had been established clinically [4]. More recent investigations suggest an important prognostic role of EMB in the diagnosis of cardiomyopathies. Felker et al. [5] followed 1,230 patients with unexplained cardiomyopathy who underwent EMB and right heart catheterization. Approximately $50 \%$ of patients demonstrated a specific cardiomyopathy, and $15 \%$ were given a specific histological diagnosis via EMB. The study demonstrated that survival in patients with myocarditis was not significantly different than patients with idiopathic cardiomyopathy. While the prognostic value of EMB in cardiomyopathy was aptly demonstrated in this study, only a small percentage of the original study population was given a specific histological diagnosis from EMB [5]. Another more recent study specifically examined long-term survival rates among 147 patients diagnosed with either fulminant or acute myocarditis [6]. Biopsied specimens were taken from the RV septum, and analyzed using the Dallas criteria (inflammatory infiltrate and associated myocyte damage not characteristic of 
ischemic event). Patients with fulminant myocarditis, while critically ill at presentation, maintained better long-term survival outcomes than patients presenting with acute myocarditis [6]. While considerable progress has been made in obtaining an EMB specimen, histological diagnosis of myocarditis still poses considerable limitations. The diagnosis of myocarditis, as described by the 1986 Dallas criteria, arguably has limitations in sampling error, discrepancies among investigator interpretation, differences in viral and immune marker expression in the myocardium, and varying degrees of response to therapy among Dallas criteria myocarditis [7].

The 2007 American Heart Association (AHA)/American College of Cardiology Foundation (ACCF)/European Society of Cardiology (ESC) scientific statement on EMB gives it a class I recommendation in the setting of an unexplained new-onset heart failure of 2-week duration with a normal-size or dilated left ventricle and hemodynamic compromise, or unexplained new-onset heart failure of 2-week to 3-month duration with a dilated left ventricle and new ventricular arrhythmia, or second- or third-degree atrioventricular block, or in patients who do not respond to usual care within 1-2 weeks [8]. The 2013 ESC Working Group on Myocardial and Pericardial Diseases position statement also supports the aforementioned recommendation [9]. However, the sensitivity of EMB for GCM decreases with duration of symptoms [10]. In a study by Kandolin et al. [11] of 72 young adult patients aged 18-55 years with initially unexplained atrioventricular block in whom GCM was found in $6 \%(n=4)$ of cases [19\% $(n=14)$ were diagnosed with cardiac sarcoidosis], EMB had a $25 \%$ diagnostic rate, comparable to that reported in a prospective GCM registry (28\%) where the criteria for biopsy were an acute cardiomyopathy complicated by heart block, ventricular arrhythmias, or lack of response to usual care $[11,12]$. The sensitivity of RV EMB is $80-85 \%$ in GCM, and its yield is significantly improved if prior site localization is done with cMRI [10, 13]. In addition, sensitivity of EMB has been shown to increase from $68 \%$ to $93 \%$ upon re-biopsy in GCM patients [11]. None of the guidelines currently recommend re-biopsy in a suspected case of acute, fulminant myocarditis where the first biopsy is inconclusive or discordant with the clinical scenario, as demonstrated by our case, where the EMB was non-diagnostic but had features suggestive of arrhythmogenic RV cardiomyopathy/dysplasia (ARVC/D). Clinically, however, there was a high index of suspicion for GCM given the history of an immune disorder in a first-degree relative, VT of right bundle branch morphology as the presenting manifestation, typical cMRI features, and fulminant clinical course despite optimal medical therapy. The value of EMB depends on the availability of expert cardiovascular pathology. Routine evaluation of EMB specimens includes formaldehyde-fixed, hematoxylin and eosin-stained sections. Depending on the clinical questions, elect samples may be sent after glutaraldehyde fixation for electron microscopy or freshly frozen (or preserved with ribonuclease inhibitors) for viral genome analysis.

Also, LV EMB, a procedure that is rarely performed in medical centers across the United States, could have provided crucial diagnostic information in our case due to predominant LV involvement. This is supported by the recently published data on safely performing LV EMB in the largest case series to date, by Chimenti and Frustaci [14]. A total of 4,221 patients underwent EMB; 1,153 underwent LV EMB, 672 RV EMB, and 2,369 both LV and RV EMB. The overall risk of major complications was 
remarkably low over the 28-year period of the study, lower with LV than RV EMB (0.33\% vs. $0.45 \%$, respectively), probably due to thinner $\mathrm{RV}$ free wall [13]. The diagnostic yield was higher with LV EMB, of disorders primarily affecting the left ventricle, compared to RV EMB (97.8\% vs. 53\%, respectively) [15]. Surprisingly enough, the diagnostic yield of LV EMB was higher for ARVC/D, a disorder that primarily affects the RV "triangle of dysplasia" [16]. Another study by Yilmaz et al. [17], comparing right and LV EMB in 755 patients with suspected myocarditis and/or non-ischemic cardiomyopathy, observed similar complication rates in left and RV EMB (0.64\% vs. $0.82 \%$, respectively). Biventricular $\mathrm{EMB}$ yielded more frequent diagnostic results (79.3\%) compared to left or right ventricleselective EMBs $(67.3 \%, P<0.001)$ [17]. In clinical scenarios in which the left ventricle if solely or primarily affected, LV biopsy may add substantially to the sensitivity of EMB for the diagnosis of myocarditis and probably GCM [18, 19].

The diagnostic accuracy can be optimized when the EMB is performed by experienced cardiac interventionalists, sampling error reduced by performing $\mathrm{EMB}$ early in the course of the disease, and taking multiple samples, at least 3 , each $1-2 \mathrm{~mm}$ in size from either the right or the left ventricle [15], and performing immunohistochemistry and viral genome amplification for assessment of suspected myocarditis [18].

\section{CONCLUSION}

Acute GCM is a life-threatening condition that requires prompt evaluation, initiation of immunosuppressive therapy and consideration for mechanical circulatory support or cardiac transplantation. EMB is pivotal to establish the correct diagnosis, and should be included in the diagnostic algorithm for fulminant myocarditis. The AHA/ACCF/ESC scientific statement on EMB does not discuss re-biopsy of the RV, or proceeding further to $\mathrm{LV}$ EMB to improve diagnostic accuracy [8]. If the results of the first RV EMB are inconclusive or discordant with the clinical scenario, then re-biopsy or LV EMB should be considered in the clinical scenario of fulminant myocarditis.

\section{ACKNOWLEDGMENTS}

No funding or sponsorship was received for this study or publication of this article. All named authors meet the ICMJE criteria for authorship for this manuscript, take responsibility for the integrity of the work as a whole, and have given final approval for the version to be published.

Conflict of interest. Ankur Kalra, Rachel Kneeland, Michael A. Samara, and Leslie T. Cooper Jr declare no conflict of interest.

Compliance with ethics guidelines. Informed consent was obtained from this patient for being included in the paper.

Open Access. This article is distributed under the terms of the Creative Commons Attribution Noncommercial License which permits any noncommercial use, distribution, and reproduction in any medium, provided the original author(s) and the source are credited.

\section{REFERENCES}

1. Cooper LT Jr. Myocarditis. N Engl J Med. 2009;360(15):1526-38. 
2. Ukena C, Mahfoud F, Kindermann I, Kandolf R, Kindermann M, Böhm M. Prognostic electrocardiographic parameters in patients with suspected myocarditis. Eur J Heart Fail. 2011;13(4):398-405.

3. Blauwet LA, Cooper LT. Idiopathic giant cell myocarditis and cardiac sarcoidosis. Heart Fail Rev. 2013;18(6):733-46.

4. Sakakibara S, Konno S. Endomyocardial biopsy. Jpn Heart J. 1962;3(69):537-43.

5. Felker GM, Thompson RE, Hare JM, et al. Underlying causes and long-term survival in patients with initially unexplained cardiomyopathy. N Engl J Med. 2000;342(15): 1077-84.

6. McCarthy RE, Boehmer JP, Hruban RH, et al. Longterm outcome of fulminant myocarditis as compared with acute (Nonfulminant) myocarditis. N Engl J Med. 2000;342(10):690-5.

7. Baughman KL. Diagnosis of myocarditis: death of Dallas criteria. Circulation. 2006;113(4):593-5.

8. Cooper LT, Baughman KL, Feldman AM, et al. The role of endomyocardial biopsy in the management of cardiovascular disease: a scientific statement from the American Heart Association, the American College of Cardiology, and the European Society of Cardiology. Circulation. 2007;116(19):2216-33.

9. Caforio AL, Pankuweit S, Arbustini E, et al. Current state of knowledge on aetiology, diagnosis, management, and therapy of myocarditis: a position statement of the European Society of Cardiology Working Group on Myocardial and Pericardial Diseases. Eur Heart J. 2013;34(33):2636-48, 2648a-2648d.

10. Shields RC, Tazelaar HD, Berry GJ, Cooper LT Jr. The role of right ventricular endomyocardial biopsy for idiopathic giant cell myocarditis. J Card Fail. 2002;8(2):74-8.
11. Kandolin R, Lehtonen J, Kupari M. Cardiac sarcoidosis and giant cell myocarditis as causes of atrioventricular block in young and middle-aged adults. Circ Arrhythm Electrophysiol. 2011;4: 303-9.

12. Cooper LT Jr, Hare JM, Tazelaar HD, et al. Usefulness of immunosuppression for giant cell myocarditis. Am J Cardiol. 2008;102:1535-9.

13. Mahrholdt H, Goedecke C, Wagner A, et al. Cardiovascular magnetic resonance assessment of human myocarditis: a comparison to histology and molecular pathology. Circulation. 2004;109(10): 1250-8.

14. Chimenti C, Frustaci A. Contribution and risks of left ventricular endomyocardial biopsy in patients with cardiomyopathies. A retrospective study over a 28-year period. Circulation. 2013;128(14):1531-41.

15. Leone O, Veinot JP, Angelini A, et al. 2011 consensus statement on endomyocardial biopsy from the Association for European Cardiovascular Pathology and the Society for Cardiovascular Pathology. Cardiovasc Pathol. 2012;21(4):245-74.

16. Basso C, Ronco F, Marcus F, et al. Quantitative assessment of endomyocardial biopsy in arrhythmogenic right ventricular cardiomyopathy/ dysplasia: an in vitro validation of diagnostic criteria. Eur Heart J. 2008;29:2760-71.

17. Yilmaz A, Kindermann I, Kindermann $\mathrm{M}$, et al. Comparative evaluation of left and right ventricular endomyocardial biopsy: differences in complication rate and diagnostic performance. Circulation. 2010;122(9):900-9.

18. Cooper LT Jr. The role of left ventricular biopsy in the management of heart disease. Circulation. 2013;128(14):1492-4.

19. Kandolin R, Lehtonen J, Salmenkibi K, et al. Diagnosis, treatment and outcome of giant cell myocarditis in the era of combined immunosuppression. Circ Heart Fail. 2013;6:15-22. 